Helmut Martin, Z. Ma, Christoph Schmittner, Bernhard Winkler, Martin Krammer, Daniel Schneider, Tiago Amorim, Georg Macher, Christian Kreiner

\title{
Combined automotive safety and security pattern engineering approach
}

Journal article | Accepted manuscript (Postprint)

This version is available at https://doi.org/10.14279/depositonce-9868

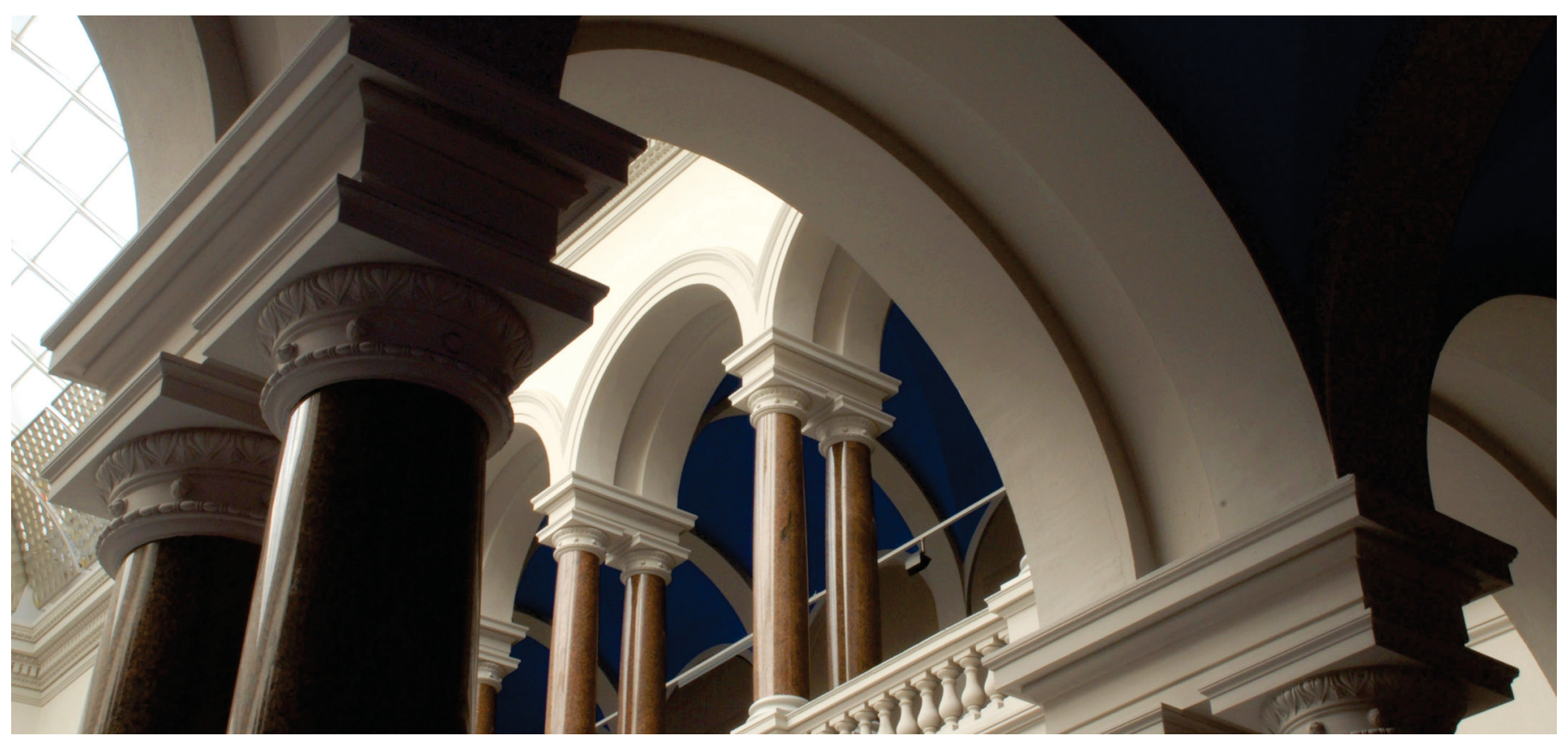

Martin, H., Ma, Z., Schmittner, C., Winkler, B., Krammer, M., Schneider, D., Amorim, T., Macher, G., \& Kreiner, C. (2020). Combined automotive safety and security pattern engineering approach. Reliability Engineering \& System Safety, 198, 106773. https://doi.org/10.1016/j.ress.2019.106773 
This is the Accepted Manuscript of: Martin, H.; Ma, Z.; Schmittner, C.; Winkler, B.; Krammer, M.; Schneider, D.; Amorim, T.; Macher, G.; Kreiner, C. (2020). Combined automotive safety and security pattern engineering approach. Reliability Engineering \& System Safety, 198, 106773. https://doi.org/10.1016/j.ress.2019.106773

This work is licensed under a Creative Commons Attribution-NonCommercial-NoDerivatives 4.0 International License, https://creativecommons.org/licenses/by-nc-nd/4.0/.

\title{
Combined Automotive Safety and Security Pattern Engineering Approach
}

\author{
H. Martin ${ }^{1}$, Z. Ma ${ }^{2}$, Ch. Schmittner ${ }^{3}$, B. Winkler ${ }^{1}$, M. Krammer ${ }^{1}$, D.

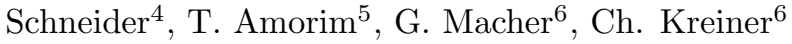 \\ Graz and Vienna, Austria - Kaiserslautern and Berlin, Germany
}

\begin{abstract}
Automotive systems will exhibit increased levels of automation as well as ever tighter integration with other vehicles, traffic infrastructure, and cloud services. From safety perspective, this can be perceived as boon or bane - it greatly increases complexity and uncertainty, but at the same time opens up new opportunities for realizing innovative safety functions. Moreover, cybersecurity becomes important as additional concern because attacks are now much more likely and severe. However, there is a lack of experience with security concerns in context of safety engineering in general and in automotive safety departments in particular. To address this problem, we propose a systematic pattern-based approach that interlinks safety and security patterns and provides guidance with respect to selection and combination of both types of patterns in context of system engineering. A combined safety and security pattern engineering workflow is proposed to provide systematic guidance to support non-expert engineers based on best practices. The application of the approach is shown and demonstrated by an automotive case study and different use case scenarios.
\end{abstract}

Keywords: ISO 26262, SAE J3061, Engineering workflow, Safety pattern, Security pattern, Automotive

\footnotetext{
${ }^{1}$ Virtual Vehicle Research Center

${ }^{2}$ AVL List GmbH

${ }^{3}$ Austrian Institute of Technology GmbH

${ }^{4}$ Fraunhofer IESE

${ }^{5}$ Technische Universität Berlin

${ }^{6}$ Graz University of Technology
} 


\section{Introduction}

Future applications in the automotive domain will be highly connected. They will rely on interacting functionalities exchanging data via various networking channels, and storing or receiving their operational data in or from the cloud. On the one hand, there is an enormous potential in these new types of cyber-physical system (CPS) applications and services, which are bound to revolutionize the automotive domain, as we know it today. On the other hand, ensuring safety and security of next generation automotive systems is a significant and comprehensive challenge that needs to be addressed before promising visions can become reality and an economic and societal success story. Today, practitioners in the automotive domain are well experienced to deal with safety aspects during the development of regular automotive systems, because a safety standard has been available since 2011. However, there is a lack of knowledge on how to handle related security aspects, which strongly gain importance given the trend towards CPS. The required security knowledge is either just non-existent, or, maybe even more often, distributed over different organizational units in a company and thus not easily accessible. Furthermore, there is no automotive specific security standard available at the moment.

Given the tight interconnection and the mutual impact of safety and security aspects, there is a need for a combined engineering approach enabling safety and security co-engineering. Moreover, given the present lack of experience in safety and security co-engineering, we think that providing additional guidance to engineers would be highly beneficial.

The safety domain has collected valuable experience in using model-based approaches for safety analysis [1] and integration of safety engineering with systems engineering by using a shared model as common viewpoint [2]. We extend this towards security and show how model-based engineering can support system, safety and security engineering.

In this paper, we specifically focus on the proper and due consideration of 
larly urgent problem related to the aforementioned challenge. Consequently, we propose a systematic pattern-based and ISO 26262-oriented approach for safety and security co-engineering in the automotive domain. Through the use of patterns, we aim to close the security knowledge gap by harvesting its manifold solutions, reuse of architectural artifacts enabled by abstraction, cross-domain exchange of solution concepts, etc. Apart from the systematic interlinking of safety and security patterns, we elaborate how these patterns can be specified and maintained.

40 for Safety and Security Co-engineering in the Automotive Domain" from Amorim et. al [3] presented at the main track of SafeComp2017 7 in Trento/Italy. The figures in the methodology part and in the battery use case are taken from that publication. For this journal paper we provide more details regarding the 45 pattern description and more insight regarding the co-engineering methodology. Furthermore, we extended the application of the methodology on the battery management system case study regarding support of pattern engineering by further use case scenarios and emphasizing Model-based Systems Engineering (MBSE).

\section{Background and Related Work}

This section provides an overview about architectural patterns in general, safety patterns, security patterns, safety and security co-engineering, and current relevant automotive guidance for safety and cybersecurity.

\subsection{Relevant Automotive Guidance for Safety and Cybersecurity}

55

ISO 26262 ("Road vehicles - Functional safety") 4 is an automotive domainspecific safety standard. It provides a structured and generic approach for the

\footnotetext{
${ }^{7}$ http://safecomp17.fbk.eu/
} 
complete safety life cycle of an automotive E/E system including design, development, production, service processes, and decommissioning. ISO 26262 recommends requirements and techniques for system, software, and hardware design to achieve functional safety for E/E systems. An Automotive Safety Integrity Level (ASIL) is used as a central metric for determination of development efforts required for the entire safety life cycle. For instance, the usage of established design patterns is recommended for all ASIL levels for each sub-phase of software development, as described in Subsection 4.4.7 in Part 6 of ISO 26262. Concerning security, the first edition, released in 2011, does not consider it explicitly neither there is any support or guidance. The second edition ISO 26262 (released by end of 2018) contains only some guidance where an interaction between safety and security may occur from a safety perspective. Part $4 \& 6$ for system and software engineering point for the requirement and architecture consolidation to the need to coordinate safety and security requirements. In addition Part 2 for the safety management requires communication channels between functional safety and disciplines related to functional safety like cybersecurity. We present here an approach which uses model-based engineering and patterns to formalize the interaction between safety and security for the system design and show an approach to address the new requirements regarding interaction.

SAE J3061 [5] is a cybersecurity process framework for secure development life cycle tailored to the automotive domain by using a corresponding V-Model, as defined in ISO 26262. In SAE J3061, safety and security interaction points were defined to coordinate the two engineering processes. In 2017 development of ISO/SAE 21434 ("Road vehicles - Cybersecurity engineering") 6] has been started as a joint project between ISO and SAE. The goal is to develop a cybersecurity engineering standard for road vehicles that includes requirements for cybersecurity process and a common language for communicating and managing cybersecurity risk among stakeholders based on ISO 26262 and SAE J3061.

85 It is planned to include similar guidance for the interaction between safety and cybersecurity from a cybersecurity perspective. 


\subsection{Safety and Security Co-Analysis and Co-Engineering}

Safety and security aspect must be considered in a holistic way due to tighter interactions between safety and security in automotive CPS. In this paper, safety \& security co-analysis refers to methods and techniques that can be used to identify safety hazards and security threats in a joint approach. Co-analysis includes activities in the early stage of the development life cycle, e.g. in the requirements engineering as well as the design phase. Safety \& security co-engineering refers to engineering activities that consider both safety and security and their interactions in the development life cycle like trade-off analysis or shared testing, verification and validation. Furthermore, co-engineering considers all phases of the life cycle, in which co-analysis is an integral part.

In the context of automotive domain, existing analysis method Hazard Analysis and Risk Management (HARA), which is standardized in ISO 26262 for safety, can be extended with Threat Analysis and Risk Assessment (TARA) method, as mentioned in SAE J3061 to identify cybersecurity risks 7]. Other proposals include Failure Mode and Vulnerability Effect Analysis (FMVEA) 8] and Security Aware Hazard Analysis and Risk Assessment (SAHARA) [9] that aim at combining both safety and security analysis in parallel. A safety and security co-engineering approach should include all engineering activities in the automotive system development life cycle according to relevant standards such as ISO 26262 and SAE J3061 based on the V-Model [10]. There are different approaches, partially supported by projects, to address and define a framework for co-engineering. One major approach is based on work done in SESAMO 8 on interaction points, which is currently refined in the AQUAS? project. The generic concept of interaction points is similar to our approach, defining points in the system engineering life cycle where engineering teams from different domains need to interact in order to build a system 11. A systematic pattern based interaction between safety and security can be considered as a interaction

\footnotetext{
${ }^{8}$ http://sesamo-project.eu

${ }^{9}$ https://aquas-project.eu
} 
point or, in the term of iterative refinement, an interaction cycle. Besides interaction point based co-engineering approaches there are also other approaches, like for example the approach which is developed in the AMASS $\$ 10$ project, which aims at a complete replacement of established development processes by an co-engineering process.

\subsection{Architectural Patterns}

Patterns are used to collect and organize solutions for similar problems with a general and universal solution. A well-known and proven solution for a specific problem is generalized so that it can be reused for similar recurring problems in other projects. Alexander describes the concept of using architecture patterns to solve similar problems in different projects 12 . The concept of patterns is used in many different domains including hardware and software. A good and very well-known reference is the book by Gamma et al. [13. (also known as the Gang of Four), which had a significant impact on making the pattern approach popular for software development. The book includes some general background and concepts as well as a collection of concrete patterns for object-oriented software design.

The state-of-the-art provides a few dozen safety architecture patterns [14] 15], with some being just a variation of simpler ones. Armoush introduced in his $\mathrm{PhD}$ thesis [14 new safety patterns and provides a collection of existing safety patterns and a characterization of the main pattern representation attributes for embedded systems patterns (e.g. Name, Type, ID, Abstract, Context, Problem, Structure,). These patterns are mostly based on the work of Douglas [16] 17] for hardware patterns and on Pullum [18] for software fault tolerance techniques brought into pattern notation for software patterns. Safety patterns usually 140 include some kind of hardware redundancy, multiple channels with voters, or sanity checks [15. They can address software or hardware issues and they allow systems to remain fully functional or to bring them to a safe state. Describing

${ }^{10}$ https://www.amass-ecsel.eu 
existing patterns, but the ones used in the presented case study, is out of the scope of this work.

Security engineering is an iterative and incremental process. Security patterns can be seen as the essence of sound security designs and best practices from an existing body of knowledge that can be used to solve security problems in new scenarios. During the security engineering process, security patterns can be used in requirements analysis and design to eliminate security flaws and provide additional information for security validation.

Security patterns have attracted the attention of both academic researchers and industry [19. The main focus of existing work is on the construction (including representation, classification, and organization) and application of security patterns. Security patterns are represented as textual templates or combined with UML models, in a hierarchically layered architecture or in a search-able pattern library. Security patterns have been proposed for requirements engineering, software system design such as web services, and Service-Oriented Architectures 20. Open Security Architecture is a community-based online repository of security control patterns based on the ISO 27000 information security standard family for enterprise IT systems, in which patterns are represented as text and graphical architecture designs in a consistent template. In recent years, security patterns have also been proposed for CPS [21].

\subsection{Model-based Systems Engineering}

Model-Based Systems Engineering (MBSE) is an efficient approach to specify, design and analsze complex systems. Estefan et. al 22] provide an overview of MBSE methodologies and cover a collection of related processes, methods, and tools used to support the discipline of systems engineering and the role of the system modeling language SysML in that context. SysML is a semi-formal, general purpose modeling language. It is based on Unified Modeling Language 170 (UML), constructed for systems engineering applications and is standardized 
by OMG 11 23. SysML concepts concern requirements, structural modeling, and behavioral constructs. New diagrams include a requirement diagram and a parametric diagram and adjustments of UML activity, class, and composite structure diagrams. The three main diagram types of SysML are requirement diagram, block definition diagrams and internal block diagrams. The requirement diagram provides cross cutting relationships between requirements and system models; the structural diagrams are block definition diagrams, internal block diagrams, package diagrams, and parametric diagrams; the behavioral diagrams are use case, state machine, activity diagrams, and sequence diagrams. 24]. We use in our approach the block definition diagram for the modeling of the system and the patterns. Model-based approaches are increasingly used for safety, this starts from safety analysis based on system models to the integration of safety artifacts into the system modeling [25]. Benefits of such an approach are the consistency between models, since there is only one system model with different views. There is also an ongoing development to extend Model-Based Systems Engineering towards security [26].

\section{Methodology}

Although patterns address specific problems, the context in which a pattern is applied influences how it should be applied. Therefore, practitioners require systematic guidance when using patterns to tackle safety and security problems, i.e. more than just a catalog of patterns is needed. We thus propose a modelbased safety and security pattern engineering workflow that aims at combining the two engineering processes for pattern identification and design and allows for the necessary interaction and balancing of safety and security concerns.

In addition, in order to support the described process in an optimal way, we introduce some specific extensions to the description of both types of pattern. These extensions focus on the links between the two engineering domains and

\footnotetext{
${ }^{11}$ http://www.omg.org/
} 
are thus a means to bridge the gap and come closer to a real co-engineering approach to support analysis and argumentation.

\subsection{Pattern Description}

With respect to safety pattern, extensions have been proposed to support engineers in adapting their assurance case argumentations after a pattern has been applied. Argumentation fragments, specified in Goal Structuring Notation (GSN), could, for instance, be provided specifically for each pattern [27, 15] .

For the presented approach we build on these safety pattern with argumentation guidance and propose to use something similar for security. However, in order to better support the interaction between safety and security and to foster co-engineering, we further enrich the descriptions with pointers to respective safety or security weak points related to the pattern and suggest application of corresponding analyses to investigate these issues and generate evidences for the argumentation. In general, patterns act as a central base of knowledge, which should be maintained based on the lessons learned during their application. On the one hand positive examples and scenarios can be referred and on the other hand negative examples, where the use of a pattern raised new and unwanted issues, should be documented as well.

Each pattern contains a general pattern description, which covers pattern name, pattern type, context, problem, forces solution, graphical representation. A generic pattern template is shown in Figure A.9

220 The safety pattern adopts the generic template, correspondingly describes the safety content and additionally augments it with respect to security aspects. Key constituents of the safety pattern are:

- GSN fragment of safety pattern 15

- General security implications (e.g. typical security properties that might interact with the pattern)

- Guidance wrt. security analysis (e.g. in a voter pattern, a promising attack vector might be going through the voter, thus analysis of the voter 
is recommended)

- GSN fragment of security implications [27]: The GSN fragment reflects the bullets above in a generic way, so that the integration of these security considerations into the argumentation is simplified/guided to a certain extent.

We design the security pattern to follow the same approach as the safety pattern, they adopt the generic template, describe the security content as well as additional information regarding potential safety implications. Key constituents of the security pattern are:

- GSN fragment of security pattern

- General safety implications (e.g. typical safety properties that might interact with the pattern such as hard real time requirements and encryption)

- Guidance wrt. safety analysis (e.g. introducing additional HW components in a functional path additional random HW failure have to be taken into account)

- GSN fragment of safety implication: The GSN fragment of the pattern reflects the bullets above in a generic way, so that the integration of the security pattern (as well as auxiliary safety analyses / evidences) is simplified/guided to a certain extent.

Appendix A of this paper provides examples of a safety pattern (see Figure A.10 and security pattern (see Figure A.11.

\subsection{Generic Combined Pattern-based Engineering Workflow}

The Generic Combined Pattern-based Engineering Workflow is the approach proposed in this paper to guide engineers selecting and applying safety and security patterns to develop safe and secure systems/products. This workflow is meant to be used in unison (and tightly integrated) with the usual safety and security engineering approaches. It therefore does not substitute established approaches but rather enhances them with further tasks. The approach is suitable to be used with existing patterns. The workflow can take place at the different 

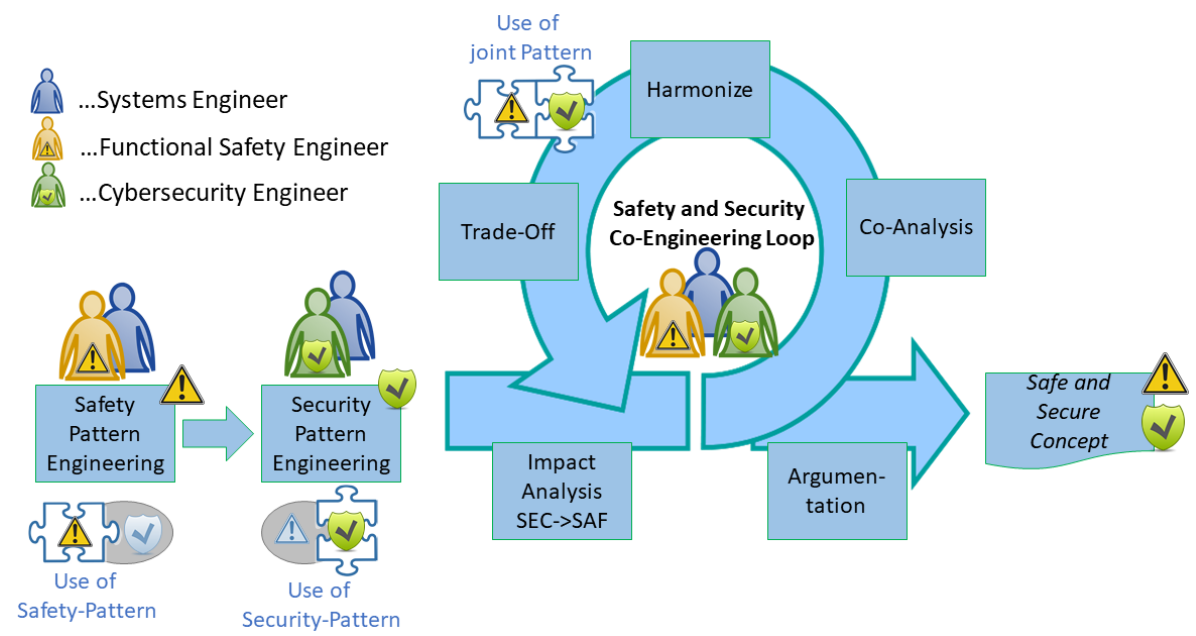

Figure 1: Generic combined pattern-based engineering workflow

development phases of the V-Model in framework of ISO 26262 [4] where design and specification are involved: concept phase, system level, hardware level and software level.

For example during the system design the Functional and Technical Concept are fully developed and both are used as input for the workflow.

The workflow is divided into three main phases happening one after the other in a waterfall fashion (cf. Figure 1): Safety Pattern Engineering, Security Pattern Engineering, and Safety and Security Co-Engineering Loop. In the first two phases specific teams are involved: Safety Pattern Engineering is performed by systems and safety engineers, and Security Pattern Engineering is performed by systems and security engineers.

The output of the workflow is then consumed by the next phases of the VModel, namely Product Development: Hardware level and Software level.

The rationale for this is that the approach explicitly focuses on security for safety (i.e., safety concerns are the main engineering drivers) and that security should start working when the first safety architecture is almost defined.

In general, further changes in the architecture might open new vulnerability 


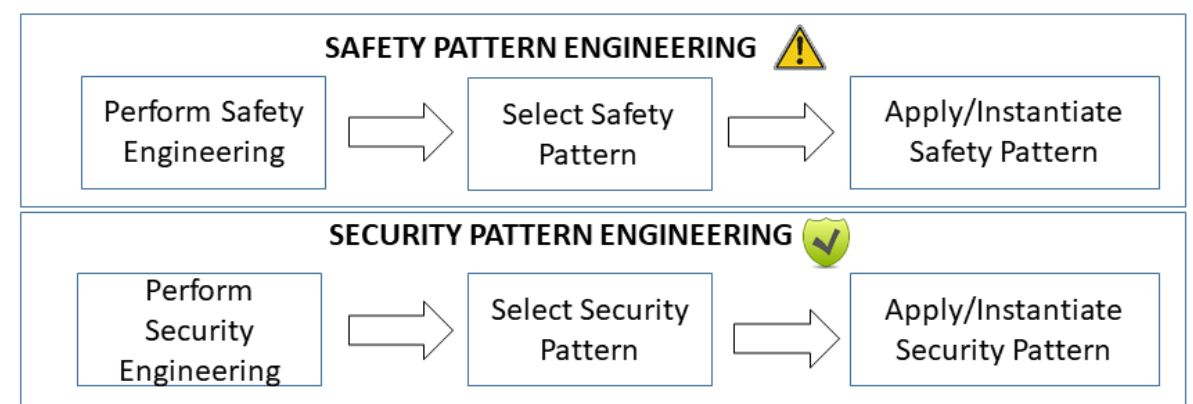

Figure 2: Safety pattern engineering and security pattern engineering tasks and the context in order to select and apply patterns appropriately. The workflow starts with established safety engineering approaches and techniques that need to be carried out until Safety Requirements (Functional or Technical) are 
available (i.e. Hazard and Risk analysis, FMEA and FTA to break down higher level to lower level requirements).

Select Safety Pattern. The decision about which pattern best fits a specific system should be analysed taking into account the problem to be addressed (typically ensuring identified Safety Requirements) and the context of the system. In the proposed pattern template, particularly the information regarding pattern type, context, problem, forces and solution are now to be considered. Besides, there are a few trade-offs that one additionally needs to take into consideration when choosing an architectural pattern, such as costs (hardware, development effort) or standardization. Current state-of-the-art [14, 16, 17] provides many patterns with detailed information about the impact in the system in the view of different dimensions (e.g. Cost, Reliability, Safety, Security), but some work is obviously required to create a comprehensive pattern catalogue that is fully in line with the templates proposed in this article. At any rate, there might always be cases that no pattern is suitable for the discovered problems and the engineer needs to come up with an ad-hoc solution.

Apply/Instantiate Safety Pattern. Instantiating an architectural pattern for a system typically implies certain adjustments to tailor the pattern towards the existing system architecture and the problem to solve. In contrast, using the pattern as-is is usually not possible, because they have been developed out of context and must thus be application and context agnostic. However, the patterns we propose (as described above and illustrated in the Appendix) provide comprehensive guidance regarding the activities to be performed as well as auxiliary material such as argumentation fragments to support the engineers in the best possible way. In addition, our safety pattern provide guidance with respect to security implications, highlighting potential weak points and providing corresponding analysis support as well as argumentation support in form of a security related GSN fragment. In summary, the pattern instantiation does not only result in a revised system architecture (now including the elements modeled by the pattern) but also in an augmented safety and security argumentation bol- 
the augmented analyses and argumentations are the prerequisites for the next task.

\subsection{Security Pattern Engineering}

In the previous phase, the architecture was updated with safety measures, safety measures have been analysed. In the Security Pattern Engineering phase, the architecture will be further analysed with regard to security vulnerabilities. Corresponding pointers might be given directly by the descriptions (security analysis and security-related GSN fragments) of the safety pattern selected in applicable security patterns. The output of this phase will be a secure architecture.

Perform Security Analysis. In this step, Security Engineering is performed on the existing system context such as functional requirements, results of Safety safety patterns. Established Security Engineering methods and techniques such as attack surface analysis, attack trees, and threat modeling can be used to identify vulnerabilities and threats. The results of this task leads to security measures that either mitigate potential threats or reduce the risks to an acceptable level. Special attention is given to vulnerabilities introduced by safety patterns.

Select Security Pattern. The security engineers should give priority to the selection of re-usable security solutions from well-established security patterns for mitigating the security risks. If multiple security patterns are available, the seSimilar to the selection of safety patterns, if no security pattern is available, an ad-hoc solution is applied. 
Apply/Instantiate Security Pattern. In this step, the instantiated security pattern is incorporated into the existing system architecture design while taking into consideration context of the system. The structure of the pattern and the instantiation approach is thereby similar to the safety pattern. In particular, beyond guidance regarding the application of the security pattern and corresponding security argumentation support, there are also pointers regarding potential safety implications which might play a role in the subsequent co-engineering loop.

\subsection{Safety and Security Co-Engineering Loop}

After the initial two phases of the Pattern Engineering Workflow, the Safety and Security Co-Engineering Loop starts. The identified patterns cover safety and security measures defined by specific requirements. Each safety and security measure should pass through the co-engineering loop to approve their adequate co-existence. Guidance with respect to the interrelationships is given by the pattern descriptions.

In this phase, lightweight versions of safety pattern engineering and security pattern engineering take place one after the other until no extra modification is required in the architecture. The fact that they are performed as a lightweight version means that the focus is on checking those aspects that experienced alteration and their respective influence on the overall system. The loop starts with the safety pattern engineering task requiring safety engineers to analyse how the newly added security patterns might impact the system safety. Some secu375 rity architecture strategy might impair, for example, the communication time between components, causing a command to arrive late. Also in this task, the results of the first security pattern engineering phase help the safety engineers to identify further points of failure that could be caused by an attack. The initial safety pattern might require some modification to add extra safety. On the other hand, if the newly proposed safety mechanisms imply new vulnerabilities or changes in the attack surface, the security engineers should detect, assess, and propose new solutions. This is what happens during the security pattern 
engineering performed in the lightweight version. This goes on like a cycle and stops when the system fulfills the desired safety and security requirements. Updating supporting documentation and updating the architecture are also tasks to be performed.

In the co-engineering loop iterative activities have been defined, which are described in the following and shown in a flow diagram in Figure 3 (Sx...Stepx, D...Decision): (S1.) Safety Impact Analysis. After successful instantiation of the security pattern in the architecture, a safety impact analysis has to be performed. This analysis should check what happens if the introduced security measures may have any safety impact. But it is not only the safety failure of the security measure, it is also the possibility that the presence of that security measure modifies the way the failures of any other elements of the architecture propagate in a modified manner, i.e. a complete safety analysis of failure modes of new/modified elements as well as redo safety analysis of other existing elements regarding any impact on existing failure modes.

(S1.D) Decision: Is there an impact of Security on Safety?

(S1.D.b) Answer: YES - Continue to with Co-Analysis

(S2.) Co-Analysis. Perform Co-Analysis for safety and security aspects by methods, such as SAHARA or FMVEA for identification of effected elements regarding new failure modes (safety) or new vulnerabilities (security). (S3.) Harmonization. Joint engineering team elaborates and defines safety and security measures based on available patterns.

(S3.D) Decision: Is harmonization successful without any side-effect between safety and security measures?

(S3.D.a) Answer: YES - A possible solution has been elaborated. All safety and security requirements are covered without any contradiction ("freedom of 


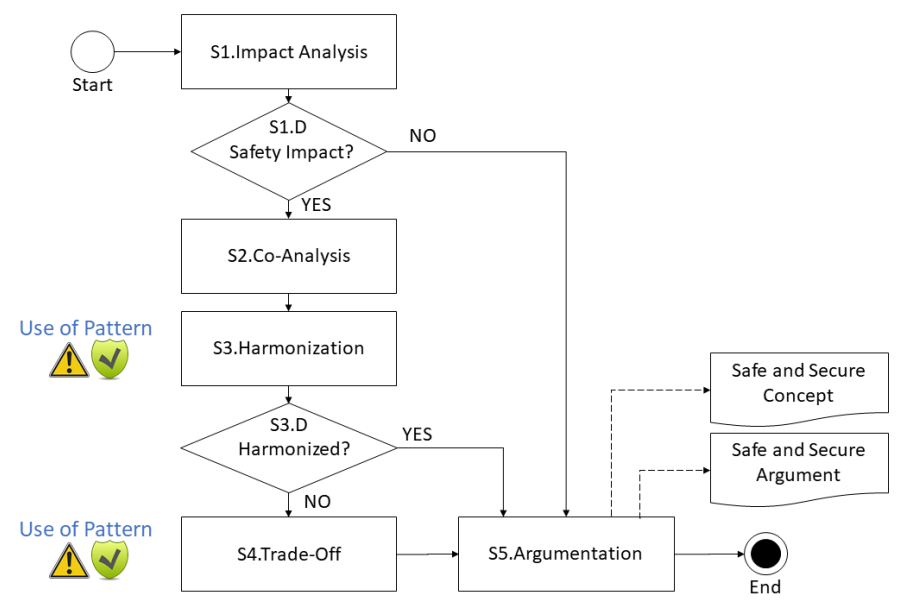

Figure 3: Flow chart of co-engineering loop iteration

interference"). Safe and Secure Concept is available. $\rightarrow$ Continue to (S5.) Argumentation.

(S3.D.b) Answer: NO - Proposals for different solutions are available. $\rightarrow$ Continue to (S4.) Trade-Off.

(S4.) Trade-Off. Joint engineering team has to prioritize which aspect is more relevant and a trade-off between safety and security measures have to be chosen. The trade-off can be supported by specific safety and security pattern, where a different pattern may be chosen to handle that issue and a compromise has to be achieved. (S5.) Argumentation. The final activity of the loop will be Argumentation. In that task the safe and secure concept will be available and all rationals will be collected to compile the final safe and secure argument of the elaborated concept, that provides a piece to the Assurance Case.

\section{Automotive Case Study} is applied to an automotive case study, namely Electrified Hybrid Powertrain 


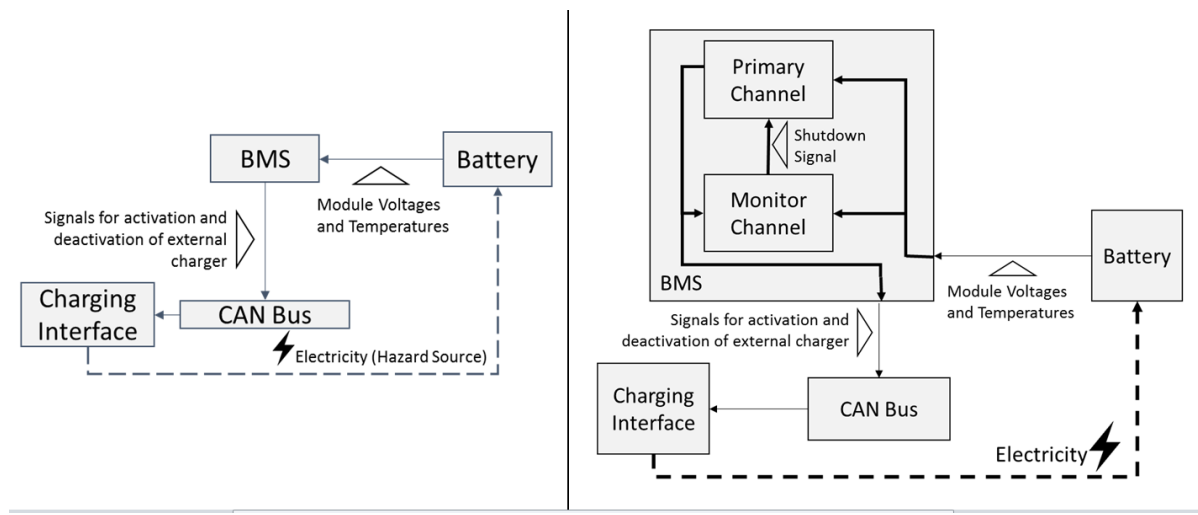

Figure 4: [Left]: Automotive Battery Use Case | [Right]: Architecture with the safety pattern applied

Battery Management System.

\subsection{Use Case Description}

Our automotive use case example of a connected electrified hybrid powertrain is a combination of one or more electric motor(s) and a conventional internal combustion engine, which is currently the most common variant of hybrid powertrains. The variety of powertrain configuration options increases the complexity of the powertrain itself as well as the required control systems, which include software functions and electronic control units. With the integration of connectivity features, further novel vehicle functionalities and new business models can be discovered. Therefore, we focus on an integral part of every connected hybrid powertrain, the battery management system (BMS), and its functionalities related to the connection to the external world; in this case especially the connections with the charging unit. In this section, we investigate a specific use case scenario of the connected hybrid powertrain: charging of the battery system by connecting it with an external charging unit. Figure 4 [Left] shows the most relevant elements: battery (including all battery modules), BMS, CAN communication, charging interface, and external charging unit. 


\subsection{Application of the Approach}

445 flow presented in the previous subsection in the use case scenario in an early development phase called "concept phase".

The described approach to cover safety activities is based on the functional safety standard ISO 26262 and in particular the parameters (e.g. severity, exposure, controllability) used in HARA are taken from ISO 26262-part3, which covers the concept phase.

For security ISO/SAE 21434 [6] is still in development and SAE J3061 was pushed back to work in progress. We use threat modeling as a well established security analysis method for the automotive domain [28, [29], 30]. In order to for Enterprise Architect (EA) 31 ${ }^{12}$. In this approach a model of the system is evaluated based on a formal description of threats to determine threats applicable to the analysed system. Different levels of the add-in are available. Furthermore, the MBSE tool EA provides good user support and possibilities rating objects or classes that can be abstracted from a general set of modeling scenarios. Therefore, patterns are considered as an excellent means of achieving re-use and building in robustness. However, pattern modeling inherently introduces front-loading principles. A library of patterns needs to be built first, in order to provide the engineer with a sufficient list of options to choose from.

\subsubsection{Safety Pattern Engineering}

Perform Safety Analysis. We describe in the following a small summary of the results of this task up to the level of Functional Safety Requirements:

470

Hazard: Wrong estimation of charging status.

Comment: The battery of electric vehicles can be very dangerous in case of

\footnotetext{
12 www.sparxsystems.eu
} 
overcharging, even causing explosions. If the charging status of a battery is estimated wrongly, extra energy might be supplied, leading to a hazardous situation.

\section{Operational situation: Parking}

Comment: The hazard will only happen while charging, and this can only be performed while the car is parked. This hazard might also occur while driving when architectures with regenerative systems are considered.

Hazard classification: Severity: 3 - Exposure: 4 - Controllability: 2

Resulting hazard ASIL: [C]

Safety goal: Estimate correct status of cycle while charging.

Safe state: "HV Battery disconnected" AND "Driver alerted".

Functional Safety requirement: Detect failures and errors from BMS.

Select Safety Pattern. The results from Safety Engineering describe two possible safe states for the system that are compliant with the Safety goal. The Disconnect HV battery measure would cut off the power supply, the source of the hazard. The Alert driver measure would issue a warning to the driver. The car will be in parking mode if the hazard occurs (operational situation: Parking); therefore, full functionality in case of fault occurrence is not required.

We should apply to the architecture a pattern that helps fulfilling the Functional Safety Requirement Detect Failure and errors from BMS. We selected the Monitor- Actuator Pattern [16] (cf. Figure 4[Right]) which provides heterogeneous redundancy. This pattern adds to the architecture a monitoring channel that detects possible faults and triggers the primary channel to enter its fail-safe state. The Monitor-Actuator Pattern is suitable to systems with low availability requirements and addresses the problem of finding an appropriate mechanism for detecting failures or errors without incurring higher costs.

Apply/Instantiate Safety Pattern. The Monitor-Actuator Pattern was instantiated as depicted in Figure 4. Only changes to the BMS component were made. 


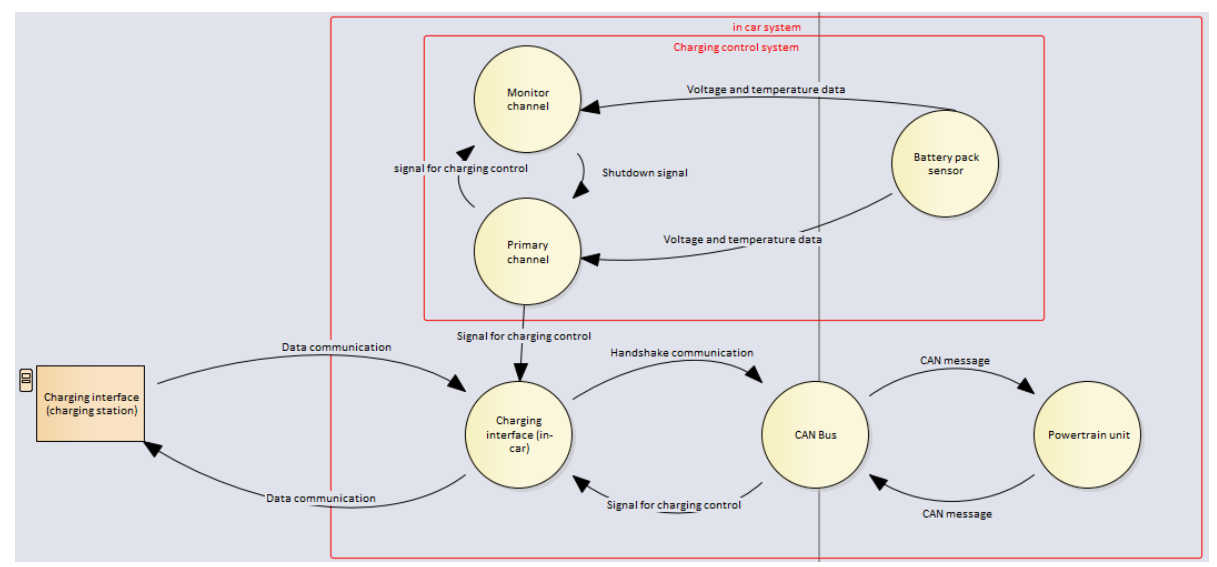

Figure 5: Threat modeling of architecture (Tool: Enterprise Architect 14 with ThreatGet Plugin 31])

\subsubsection{Security Pattern Engineering}

Perform Security Analysis. In this context, Security Engineering follows the initial definition of a safety pattern to identify potential security threats and vulnerabilities in the design, and to assess the risks in order to find appropriate countermeasures and apply corresponding security patterns. In this example, we use the threat modeling methodology [32, in which a system is modeled in a Data Flow Diagram (DFD). When modeling the functional blocks from the safety pattern (cf. Figure 4 in a DFD, a few transitions and extrapolations occur.

First, since threat modeling assumes that attacks happen when data flow from one process (i.e., a software component that takes input and either produces output or performs an action) to another, the logic signal flows in the safety pattern need to be translated into directional data flows according to the software architecture implementing this safety logic. Therefore, additional components are added such as the CAN bus process, which represents the communication bus in the in-car system. Second, the trust boundaries need to be defined in the DFD in order to identify attacks originating from data flows across trust boundaries. As a result, the Charging Interface (CI) is split into two 

interface on the charging station is modeled as an external interactor outside the In-car system trust boundary. There can be different levels of trust boundaries. In this case, we assume that attacks can only originate from outside the In-car system boundary. Third, at the system level, security has an influence on between the primary and monitor channel and the CI goes through the CAN bus, and the powertrain unit is connected to the same bus, the security of the CI also influences the security of the powertrain unit. Thus even though the two safety modules cannot be attacked directly due to the unidirectional data to attack the powertrain unit. Figure 5 shows the modeled architecture in DFD using the free cyber-security modelling addin for Enterprise Architect [31.

Asset: BMS software and its related functions and messages on the CAN bus are the main assets

Threat: Attack CAN bus through in-car CI

Comment: The communications from the external CI to the CAN bus is responsible for establishing and maintaining communications for charging control. An attacker can use the in-car CI as an entry point by compromising the external CI or tampering with the communications between the interfaces to inject malicious content into the CAN bus.

Impact: Safety impact of the identified threats on the assets.

Comment: The safety analysis defines the initial scope of the security analysis. The impact analysis links the results from safety and security analysis. Table 1 gives an example of the outcome of the security impact analysis. The co-analysis provides grounds for detailed security engineering requirements on the system.

Security requirements: The security requirements are related to authentication, authorization, non-repudiation, accountability, data integrity, confidentiality, privacy, and availability, which need to be further allocated to system and component level during the design. In this example, we skip the assessment 
Table 1: Security impact analysis

\begin{tabular}{|c|c|c|}
\hline Asset & Security impact & Safety Impact \\
\hline $\begin{array}{l}\text { BMS soft- } \\
\text { ware }\end{array}$ & $\begin{array}{l}\text { Integrity of BMS soft- } \\
\text { ware }\end{array}$ & $\begin{array}{l}\text { Overcharging battery } \\
\text { system }\end{array}$ \\
\hline $\begin{array}{l}\text { CAN bus } \\
\text { communica- } \\
\text { tion }\end{array}$ & $\begin{array}{l}\text { Availability of CAN } \\
\text { messages to their in- } \\
\text { tended ECUs }\end{array}$ & $\begin{array}{l}\text { Disturbing ECU func- } \\
\text { tioning on the power- } \\
\text { train system }\end{array}$ \\
\hline
\end{tabular}

security requirements.

Select Security Pattern. During security design, other factors beside requirements such as available solutions, performance and cost need to be taken into consideration. Since current CAN does not support encrypted and authenticated communication, one possible solution is to add a security gateway between the external unit and the internal CAN bus as shown in Figure 6. The security gateway is the application of the security pattern "firewall" that is placed between an unprotected internal network and untrusted external entities when communication to the outside is inevitable (see Figure A.11). The firewall pattern is a basic security measure that controls incoming and outgoing network connections between a protected and an untrusted network. It provides network access control that restricts which hosts can be accessed on the internal network. Note that other security patterns can be applied as well. For example, we might apply the "security proxy" pattern, in which an entity on the communication path between the charging station and the BMS software will not only translate different communication protocols (e.g. Ethernet to CAN), but also authenticate the charging station and verify the validity of the communication. As a repeatable solution, the security gateway is not limited to the CI. It can be applied to any communication between the CAN bus and untrusted external devices. In general, the gateway controls the network access to the internal ECUs according to predefined security policies and can also inspect 


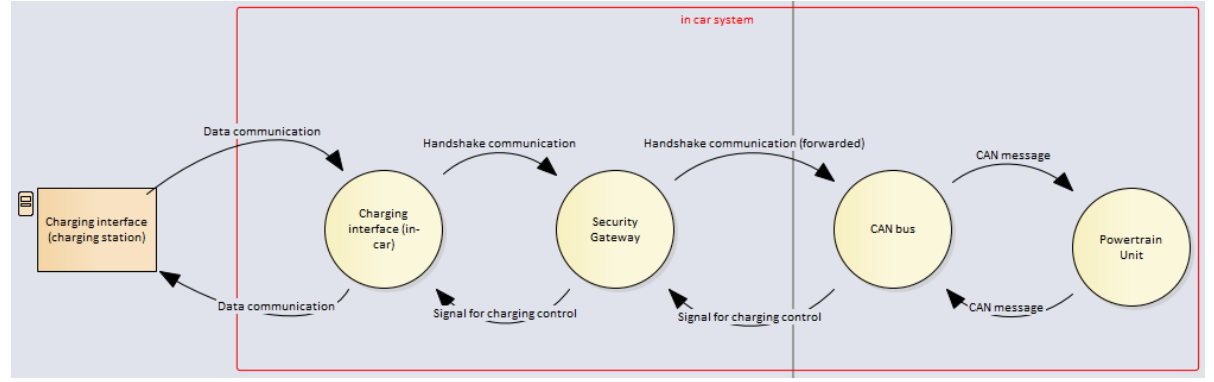

Figure 6: Security Gateway as a security pattern (Tool: Enterprise Architect 14 with ThreatGet Plugin [31]

packet content to detect intrusion attempts and anomalies. In this way, it adds security protection and segments the system without fundamentally changing the existing in-car system architecture.

Select/Instantiate Security Pattern. In Figure 6, we see the altered architecture with the Security Gateway module.

\subsubsection{Safety and Security Co-Engineering Loop}

Beyond the many benefits, a security gateway might introduce latency into the communication or block critical messages due to false-positives, which is a subject of safety impact analysis.

Safety Impact Analysis. With the inputs from previous tasks we perform a HAZard and Operability Study (HAZOP) analysis to identify potential anomalies in the provision of the service controlling the CI (cf. Table 2). The focus is thus on the changes performed to the architecture by the security engineers.

Based on the analysis we identified failure modes Omission and Late as potential causes of a hazard (cf. Table 2). Other potential failure modes are not relevant for this scenario. As input from the Security Pattern Engineering phase, we get the information that the Security Gateway adds a small latency to the communication between the CI and the BMS. This small delay can cause a minor amount of extra charging in the battery which is not a source of hazard and need no further investigation in the safety and security co-engineering loop. 


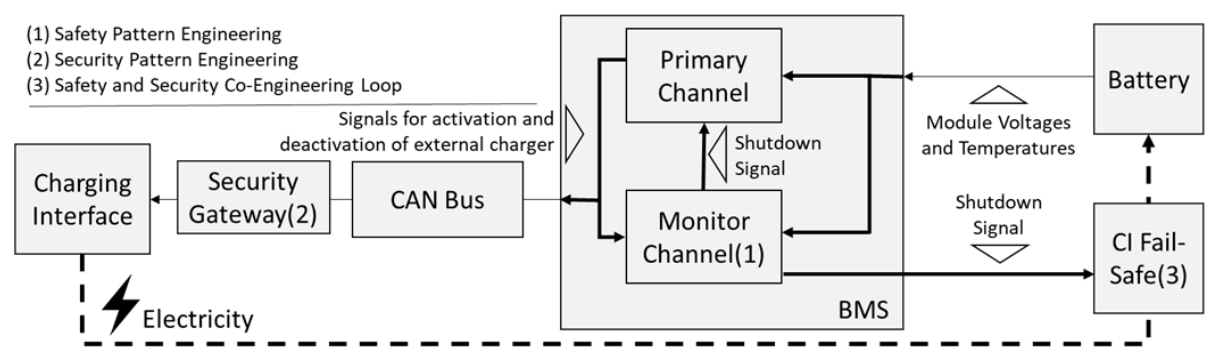

Figure 7: Architecture after the first iteration of safety and security co-engineering

Table 2: HAZOP Guideword analysis of the architecture

\begin{tabular}{l|l|l} 
Guideword & Possible causes & Possible consequences \\
\hline Commission & - & - \\
\hline Omission & $\begin{array}{l}\text { Gateway blocks message to } \\
\text { stop charging - Message gets }\end{array}$ & $\begin{array}{l}\text { Charging Interface keeps pro- } \\
\text { viding energy to battery }\end{array}$ \\
\hline Early & - & \\
\hline Late & $\begin{array}{l}\text { Additional processing time } \\
\text { slows down reaction time of }\end{array}$ & Late disconnection of battery \\
\hline Value High & - & \\
\hline Value Low & - & -
\end{tabular}


From the input received from the previous phase, we also discovered that the safety functions on the CI will not suffice in the case of a hacker attack. This need a further investigation by performing a Safety and Security Analysis. Safety and Security Co-Analysis. The two-stage SAHARA method then combines the outcome of the security analysis with the outcomes of the safety analysis. The SAHARA method applies a key concept of the HARA approach, the definition of ASILs, to the STRIDE analysis outcomes. Security threats that might lead to a violation of safety goals can be handed over to HARA for further safety analysis. This helps to improve completeness of safety analysis in terms of the safety requirement of analysis of 'foreseeable misuse', in this case hazardous events initiated due to security attacks. For the battery system it can be seen that security hazard are aiming a overloading of the battery because the CI keeps providing energy to the battery.

Harmonize. To tackle this issue a CI fail-safe device connected to the Monitor channel was integrated (cf. Figure 7). Of course, one obvious drawback in this solution is the extra cost incurred due to extra hardware and installation. The changes in the architecture neither create new vulnerabilities nor jeopardize the current mechanisms already in place. Furthermore, additional requirements can be added to the Security Gateway in the security concept, specifying that firewall rules shall not block critical messages in charging process, which can be implemented and tested in the development phase.

Trade-Off. After finalization of the safety and security pattern engineering activities, the design can be reviewed to check whether all applied patterns can co-exist and whether there is no unwanted influence. While there is a direct review of the design with the applied patterns after each iteration, a final check can ensure the soundness of the design. In [33] we presented a quantitative methodology for security risk assessment by combining FMVEA and SAHARA methods with the FAIR method. This enables the analysis of security and failure event chains, as well as a coordinated risk management for safety and 
security features. In this case, it was decided to add the Security Gateway as an additional component in the system, to not only ensure that safety pattern and the security pattern do not interfere with each other, but also to support the maintainability of the security solution. Updates to the gateway do not impact the safety pattern directly.

Argumentation. During the task argumentation the safety and security concept is finally available and all argument fragments through the overall workflow are consolidated: GSN argumentation fragments of the chosen patterns (e.g. security pattern for firewall in Figure A.11), co-analysis results, and evidences of the elaborated safety and security measures from harmonization and/or tradeoff. The joint assurance case contains the combined safety and security case.

\subsubsection{Modeling of the System}

For the item definition of the system we used the block definition diagram (bdd) of SysML. The blocks, their hierarchy and multiplicities (e.g. exactly one module consists of at least one cell) are defined based on the use case description and requirements (cf. Figure $8[$ Top] $)$. Furthermore, all input and output ports are defined as known so far. This item definition is compliant to ISO 26262 and subject to further safety and security analyses.

\subsubsection{Modeling Patterns}

640

In a similar way, patterns are modeled. Patterns may contain UML classes (or blocks in SysML, as equivalent) and their relationships (cf. Figure 8 [Bottom]). The diagram is saved as a pattern and stored in a separate XML file format and stored in the pattern repository. A specific save dialog offers several options (e.g. specification of name, file-name, and category). The classes of the patterns may be replaced with classes from your system model during the integration later on, which needs to be enabled by ticking the boxes in the corresponding columns (create/merge/instance/type). To give an example, the merge option replaces an existing model element with an element defined in 


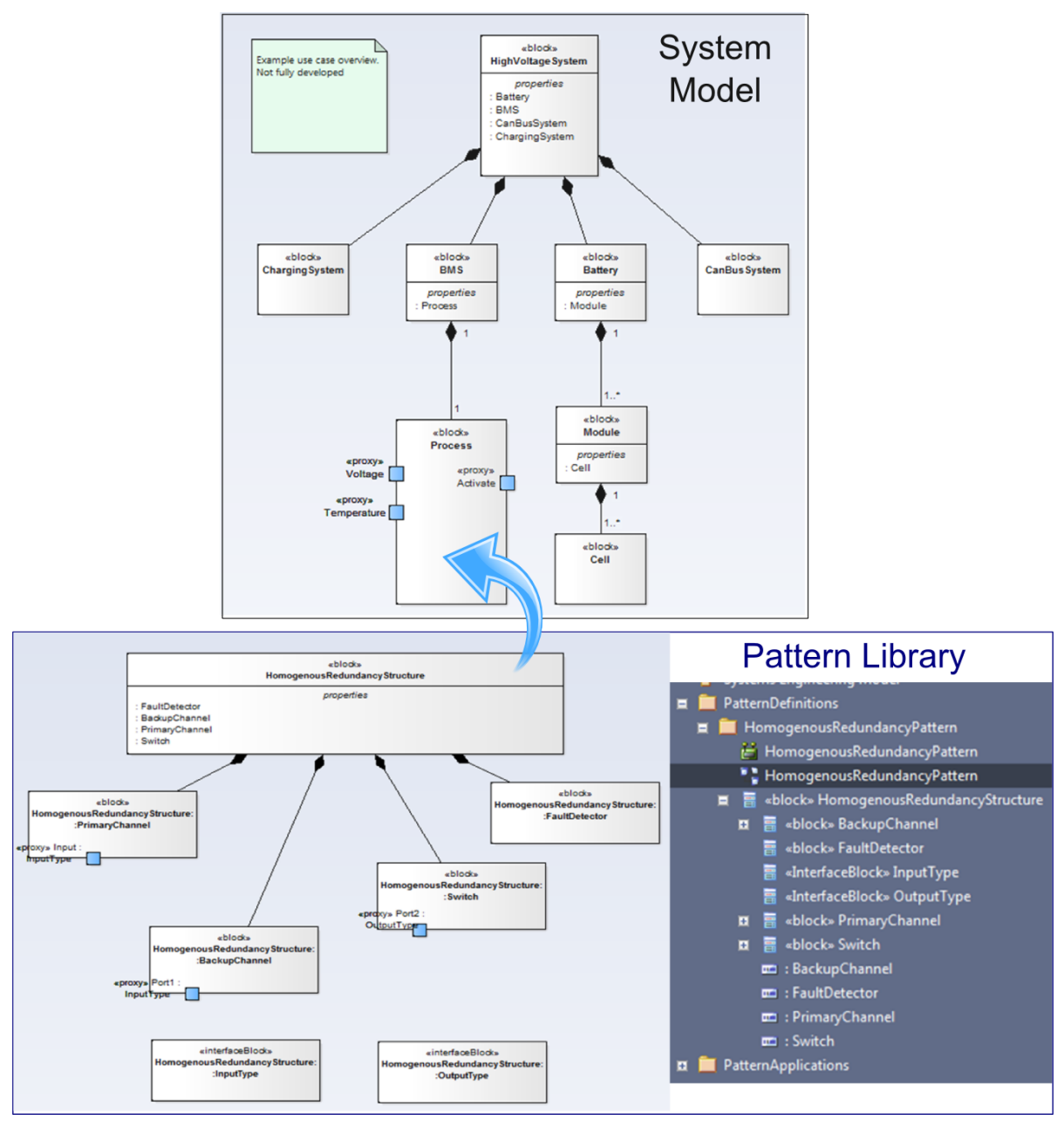

Figure 8: [Top]: Item Definition in SysML | [Bottom]: Creation of Safety Pattern [Tool:Enterprise Architect] 
the pattern. This is especially useful for larger patterns, and ensures seamless

\subsubsection{Pattern Instantiation}

The pattern may then be applied to the system model by drag and drop of the pattern to the diagram by a following dialog window, where it is possible to assign existing elements to the pattern (cf. Figure 8 Bottom). In the exam-

Pattern "HomogenousRedundancyStructure" is mapped to the BMS. Based on the mapping the tool updates the bdd with the missing classes from the chosen pattern and the pattern import is completed.

\subsubsection{Interaction with Analysis Tool}

660

Threat Modeling is a wide spread security analysis, which is also one of the contributions for co-analysis techniques like SAHARA or FMVEA. The main tool for Threat Modeling has been a plug-in for MS Visio, which only provides a graphical modeling support. The tool from AIT in development called "ThreatGet" integrates Threat Modeling into Enterprise Architect. With the integration into EA the threat modeling is based on a SysML model and resulting threats can be integrated into the workflow and guarantee traceability.

\subsection{Lessons Learned}

The definition of patterns in the EA tool is done via simple UML class diagrams and works for SysML blocks as well. The pattern are saved in separate easily a "library of patterns". Furthermore, it is easy to apply the patterns to the system model via drag and drop. The pattern mechanism of the EA tool seems to work at this stage for bdd diagrams only, which means that the pattern "just reminds you to add needed blocks" and the engineer has to connect them as needed according to the specific context. The concrete interconnections between blocks, normally realized via interfaces and ports, are out of scope of the EA patterns. This is due to the fact that at this point in time the exact 
definition of ports and interfaces, etc. are unknown. The described approach using EA limits the application of patterns to early development phases.

Currently vehicle chargers can be mainly classified into: AC charging that uses residential power outlet, DC fast charging (DCFC) that charges a vehicle by DC in much shorter time, and inductive charging that eliminate the use of cables. All of them require certain message exchange between the charging station and the vehicle during the charging process to regulate the current provided to the vehicle. SAE recommendations are often used in the industry to implement such communications on top of the physical and data link layer. For example, SAE J2847/2 "Communication between plug-in vehicles and off-board DC chargers" 34 defines message format for DC charging. These messages might be manipulated to sabotage the charging process that leads to battery explosion. Therefore, in SAE J2931/7 "Security for plug-in electric vehicle communications" [35] a set of security requirements are specified for different stakeholders involved in the system. On the other hand, standards such as ISO 17409:2015 "Preview Electrically propelled road vehicles - Connection to an external electric power supply - Safety requirements" [36] specifies the safety requirements. The safety and security pattern described here has been instantiated in certain degree in actual vehicles, which use a gateway to avoid direct data connection to charging station and relays controlled by BMS to avoid overcharging. Therefore, safety and security patterns can be an repeatable and efficient way to address and enforce safety and security requirements from related standards in system development based on industry best practices.

\section{Discussion and Conclusion}

This paper focused on the selection, combination, and application of safety and security patterns for automotive system engineering. The introduction of the Combined Pattern-based Engineering Workflow provides a systematic and 
integrated way of safety- and security-related pattern engineering. It provides comprehensive guidance and packaged knowledge with respect to the integration, as well as auxiliary existing work products, such as the (initial) results of safety and security analyses and argumentation fragments. The availability

of recurring process steps based on automotive industry standards results in faster and cheaper product development while fulfilling the need for intangible product properties, namely safety and security. The Safety and Security Co-Engineering Loops as well as the correlated guidance provided by our augmented pattern descriptions help to align the required activities systematically and foster a tight integration of safety- and security-related process steps. For instance, this means if a safety (architectural) pattern will be selected to address a specific safety requirement, additional information and guidance with respect to correlated core aspect from a security point of view are provided. A security pattern, in turn, can have a safety impact, which is again explicitly specified.

With the presented approach, the decision which pattern fits best for a specific system can be done more systematically (particularly by non-expert engineers) and taking into account the problem to be addressed and the context of the system. In general, safety and security engineering are very closely related disciplines and their synergy can be fostered when their similarities are recognized and adequate interactions are established correctly.

To demonstrate the benefits of the presented approach an automotive case study demonstrated the practical realization of our approach: Architecture of an automotive battery system was described in a semi-formal way, including identification of its main components, physical interconnections, and flows of information. The use case has been reduced in complexity for illustration purposes and it highlights the benefits of applying the Pattern Engineering Workflow for the concept phase development.

With the presented approach, we aimed to benefit from the typical strong points of patterns to overcome the lack of general security knowledge as well as organizational weak points (i.e. no integrated safety-security teams, distributed or lacking security knowledge etc) that are prevalent in the automotive domain. 
It is a promising approach that should help accelerating the application of adequate safety and security co-engineering in industry. In particular, we believe the type of pattern we introduced constitute a way to remediate the lack of security knowledge and facilitate easier and more informed integration of these two separate yet interfering disciplines. Additionally, initial positive experiences were shown, which were gained by a tool supported model-based systems engineering application, where patterns were created and applied for a specific automotive use case scenario.

\section{Acknowledgment}

Dedicated to our co-author late Christian Kreiner, who was impressive for many reasons and has been a wonderful teacher, co-worker, leader and friend. You have been everything one could look for in a good mentor, the true Master Yoda, and made working with you an exciting, inspiring and memorable experience. We will always be grateful to you for your support and kindness. May the force still be with you.

Research leading to these results has received funding from the EU ECSEL Joint Undertaking under grant agreement no. 692474 (project AMASS), EU ECSEL JU project SCOTT (no. 737422), EU Horizon 2020 research and innovation programme under grant agreement no. 732242 (project DEIS), and from the COMET K2 - Competence Centres for Excellent Technologies Programme of the Austrian Federal Ministry for Transport, Innovation and Technology (bmvit), the Austrian Federal Ministry of Science, Research and Economy (bmwfw), the Austrian Research Promotion Agency (FFG), the Province of Styria, and the Styrian Business Promotion Agency (SFG), the German Federal Ministry of Education and Research (BMBF), grant CrESt, 01IS16043. 


\section{List of Abbreviation}

Table 3: List of Abbreviation

\begin{tabular}{|l|l|l|l|}
\hline \multicolumn{4}{|c|}{ Table 3: List of Abbreviation } \\
\hline Abbrev. & Definition & Abbrev. & Definition \\
\hline AC & Alternating Current & HARA & Hazard Analysis and Risk Management \\
\hline ASIL & Automotive Safety Integrity Level & HAZOP & HAZard and OPerability Study \\
\hline BMS & Battery Management System & HV & High Voltage \\
\hline C & Controlability & ID & Identifier \\
\hline CAN & Controller Area Network & ISO & $\begin{array}{l}\text { International Organization for Standard- } \\
\text { ization }\end{array}$ \\
\hline CI & Charging Interface & IT & Information Technology \\
\hline CPS & Cyber-Physical System & MBSE & Model-Based Systems Engineering \\
\hline DCFC & Direct Current Fast Charging & MS & MicroSoft \\
\hline DFD & Data Flow Diagram & S & Severity \\
\hline E & Exposure & SAE & Society of Automotive Engineers \\
\hline E/E & Electric and/or Electronic & SAHARA & $\begin{array}{l}\text { Security Aware Hazard Analysis and Risk } \\
\text { Assessment }\end{array}$ \\
\hline EA & Enterprise Architect & STRIDE & $\begin{array}{l}\text { Spoofing, Tampering, Repudiation, Infor- } \\
\text { mation disclosure, Denial of Service, Ele- } \\
\text { vation }\end{array}$ \\
\hline ECU & Electronic Control Unit & System Modelling Language \\
\hline FDIS & Final Draft International Standard & TARA & Threat Analysis and Risk Assessment \\
\hline FMVEA & $\begin{array}{l}\text { Failure Mode and Vulnerability Eect Anal- } \\
\text { ysis }\end{array}$ & UML & Unified Modelling Language \\
\hline GSN & Goal Structuring Notation & XML & Extensible Markup Language \\
\hline
\end{tabular}

\section{References}

[1] A. Joshi, M. P. Heimdahl, S. P. Miller, M. W. Whalen, Model-Based Safety Analysis, Tech. Rep. CR-2006-213953, NASA (2006).

[2] B. Kaiser, V. Klaas, S. Schulz, C. Herbst, P. Lascych, Integrating system modelling with safety activities, in: SAFECOMP'10 Proceedings of the 29th international conference on Computer safety, reliability, and security, Springer, 2010, pp. 452-465. 978-3-642-15651-9_33

[3] T. Amorim, H. Martin, Z. Ma, C. Schmittner, D. Schneider, G. Macher, B. Winkler, M. Krammer, C. Kreiner, Systematic pattern approach for safety and security co-engineering in the automotive domain, in: International Conference on Computer Safety, Reliability, and Security, Springer, 2017, pp. 329-342. doi:10.1007/978-3-319-66266-4_22. 
[4] ISO, ISO 26262 Road vehicles - Functional safety (2011).

[5] SAE, SAE J3061 Cybersecurity Guidebook for Cyber-Physical Vehicle Systems (2016).

[6] C. Schmittner, G. Griessnig, Z. Ma, Status of the development of iso/sae 21434, in: European Conference on Software Process Improvement, Springer, 2018, pp. 504-513.

[7] G. Macher, E. Armengaud, C. Kreiner, E. Brenner, C. Schmittner, Z. Ma, H. Martin, M. Krammer, Integration of security in the development lifecycle of dependable automotive cps, in: Handbook of Research for CyberPhysical Systems Ubiquity, IGI Global, 2017.

[8] C. Schmittner, Z. Ma, E. Schoitsch, T. Gruber, A case study of fmvea and chassis as safety and security co-analysis method for automotive cyberphysical systems, in: Proceedings of the 1st ACM Workshop on CyberPhysical System Security, CPSS '15, ACM, New York, NY, USA, 2015, pp. 69-80. doi:10.1145/2732198.2732204.

[9] G. Macher, H. Sporer, R. Berlach, E. Armengaud, C. Kreiner, Sahara: A security-aware hazard and risk analysis method, in: 2015 Design, Automation Test in Europe Conference Exhibition (DATE), 2015, pp. 621-624. doi:10.7873/DATE.2015.0622.

[10] C. Schmittner, Z. Ma, T. Gruber, E. Schoitsch, Safety and security coengineering of connected, intelligent, and automated vehicles, ERCIM News 109 (2017) 23-24.

[11] T. Gruber, C. Schmittner, M. Matschnig, B. Fischer, Co-engineering-inthe-loop, Computer Safety, Reliability, and Security (2018) 151.

[12] C. Alexander, S. Ishikawa, M. Silverstein, M. Jacobson, I. Fiksdahl-King, S. Angel, A pattern language (1977). 
[13] E. Gamma, R. Helm, R. Johnson, J. Vlissides, Design Patterns: Elements of Reusable Object-oriented Software, Addison-Wesley Longman Publishing Co., Inc., Boston, MA, USA, 1995.

[14] A. Armoush, Design patterns for safety-critical embedded systems., Ph.D. thesis, RWTH Aachen University (2010).

[15] C. Preschern, N. Kajtazovic, C. Kreiner, Building a safety architecture pattern system, in: Proceedings of the 18th European Conference on Pattern Languages of Program, EuroPLoP '13, ACM, New York, NY, USA, 2015, pp. 17:1-17:55. doi:10.1145/2739011.2739028. URL http://doi .acm . org/10.1145/2739011.2739028

[16] B. P. Douglass, Real-Time Design Patterns: Robust Scalable Architecture for Real-Time Systems, Addison-Wesley Longman Publishing Co., Inc., Boston, MA, USA, 2002.

[17] B. P. Douglass, Design Patterns for Embedded Systems in C: An Embedded Software Engineering Toolkit, 1st Edition, Newnes, Newton, MA, USA, 2010.

[18] L. L. Pullum, Software Fault Tolerance Techniques and Implementation, Artech House, Inc., Norwood, MA, USA, 2001.

[19] M. Schumacher, Security engineering with patterns: origins, theoretical models, and new applications, Vol. 2754, Springer Science \& Business Media, 2003.

[20] N. A. Delessy, E. B. Fernandez, A pattern-driven security process for soa applications, in: 2008 Third International Conference on Availability, Reliability and Security, 2008, pp. 416-421. doi:10.1109/ARES.2008.89.

[21] N. E. Petroulakis, G. Spanoudakis, I. G. Askoxylakis, A. Miaoudakis, A. Traganitis, A pattern-based approach for designing reliable cyberphysical systems, in: 2015 IEEE Global Communications Conference (GLOBECOM), 2015, pp. 1-6. doi:10.1109/GLOCOM.2015.7417794. 
[22] J. A. Estefan, et al., Survey of model-based systems engineering (mbse) methodologies, Incose MBSE Focus Group 25 (8) (2007) 1-12.

[23] OMG Systems Modeling Language (OMG SysML) (2012), http://www.omg.org/spec/SysML/1.3/ (Jun. 2012).

[24] S. Friedenthal, A. Moore, R. Steiner, A practical guide to SysML: The systems modeling language, Morgan Kaufmann, 2014.

[25] G. Biggs, T. Sakamoto, T. Kotoku, A profile and tool for modelling safety information with design information in SysML, Software \& Systems Modeling 15 (1) (2016) 147-178. doi:10.1007/s10270-014-0400-x.

URL http://link.springer.com/10.1007/s10270-014-0400-x

[26] Muhammad Sabir Idrees, Yves Roudier, Ludovic Apvrille, A Framework Towards the Efficient Identification and Modeling of Security Requirements, 2010.

[27] C. Preschern, N. Kajtazovic, C. Kreiner, Security analysis of safety patterns, in: Proceedings of the 20th Conference on Pattern Languages of Programs, The Hillside Group, 2013, p. 12.

[28] A. Karahasanovic, P. Kleberger, M. Almgren, Adapting threat modeling methods for the automotive industry, in: Proceedings of the 15th ESCAR Conference, 2017, pp. 1-10.

[29] Z. Ma, C. Schmittner, Threat modeling for automotive security analysis, Advanced Science and Technology Letters 139 (2016) 333-339.

[30] M. Hamad, M. Nolte, V. Prevelakis, Towards comprehensive threat modeling for vehicles, in: the 1st Workshop on Security and Dependability of Critical Embedded Real-Time Systems, 2016, p. 31.

[31] Sparx Services CE - Cyber Security Modeling - Security by design, [Online; accessed 5. Jul. 2019] (Jul 2019).

URL https://cybersecurity.sparxservices.eu 
[32] A. Shostack, Threat Modeling: Designing for Security, Wiley, 2014.

URL https://books.google.de/books?id=asPDAgAAQBAJ

860

865

\section{Appendix A. Pattern Examples}

This section shows some pattern examples of our approach to describe pattern:

- pattern template see Figure A.9.

- safety pattern example see Figure A.10

875 


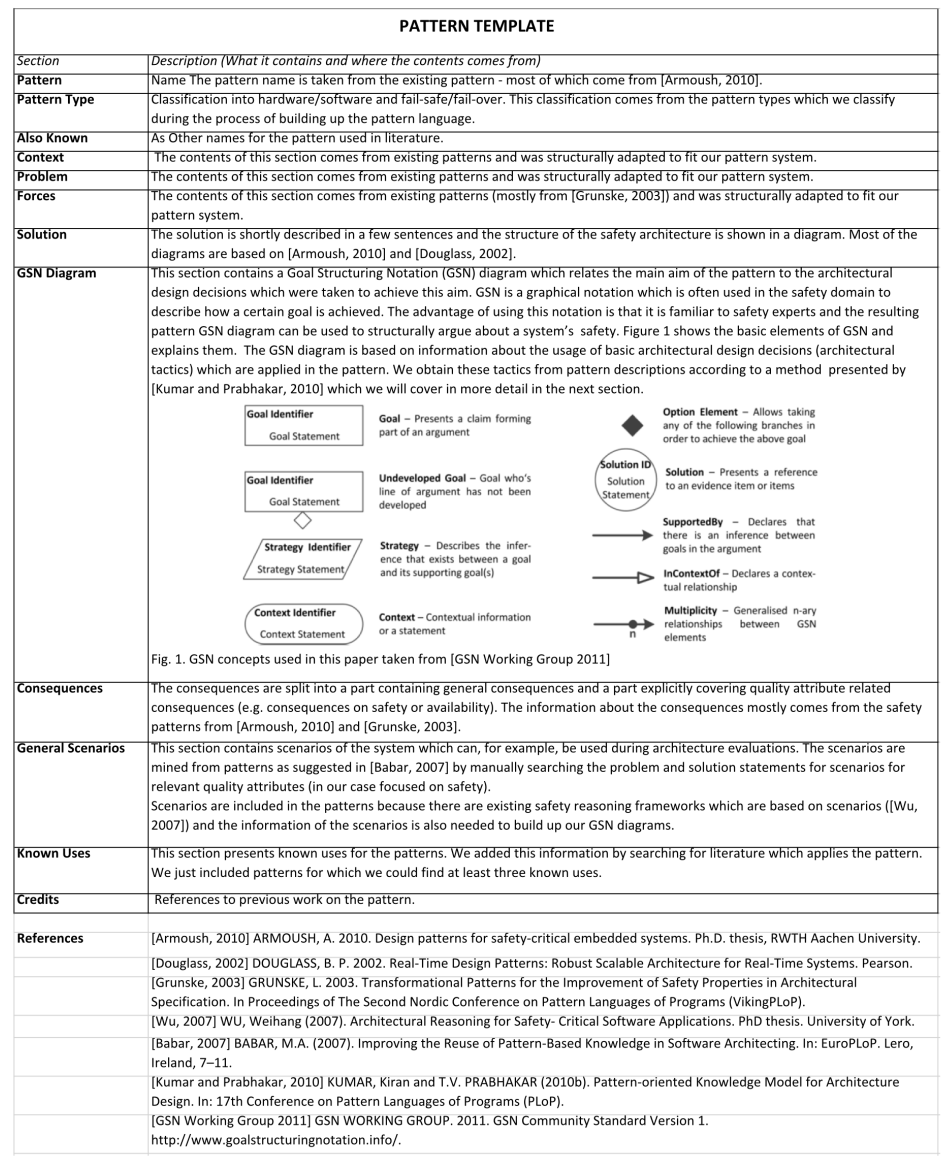

Figure A.9: Pattern Template 


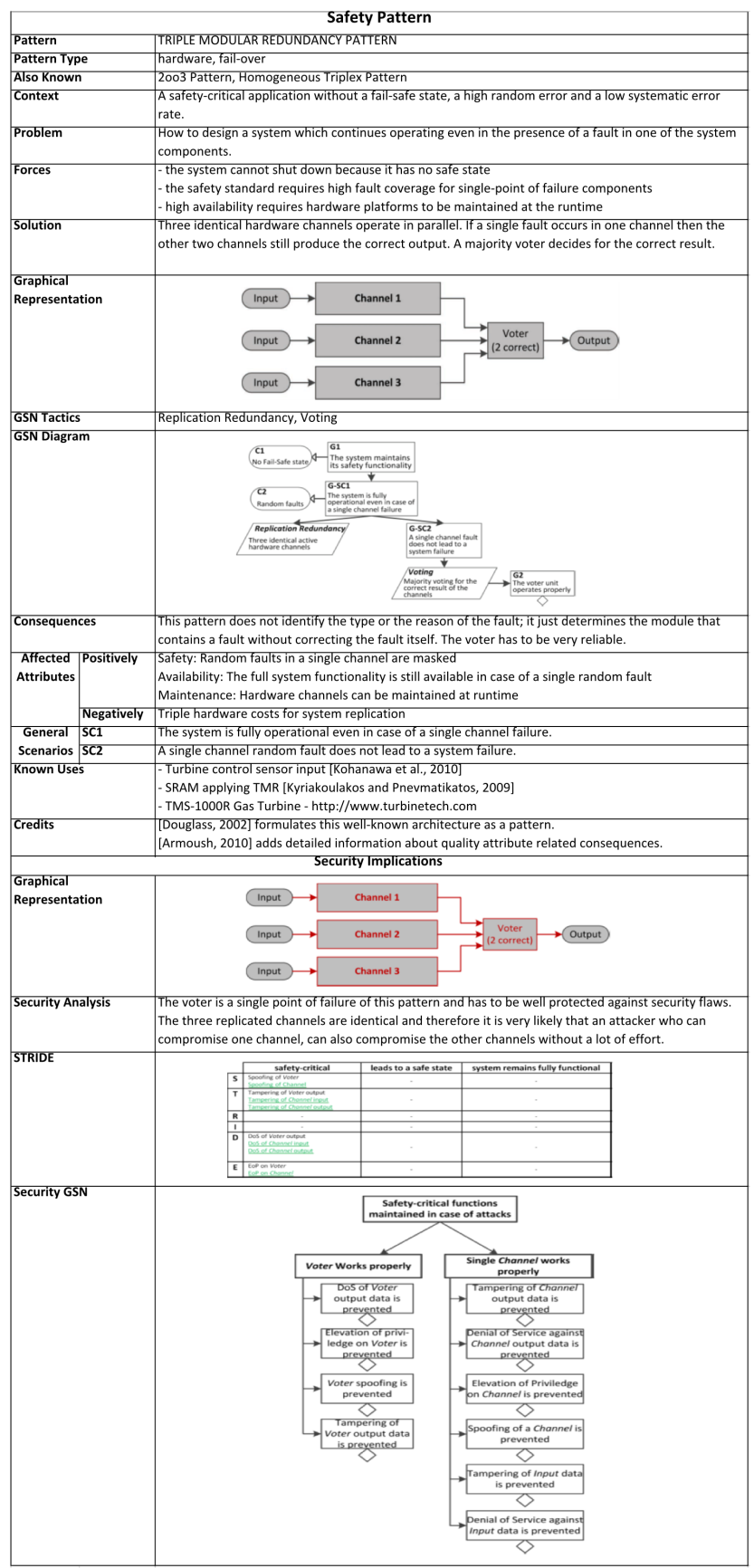

Figure A.10: Safety Pattern Example 


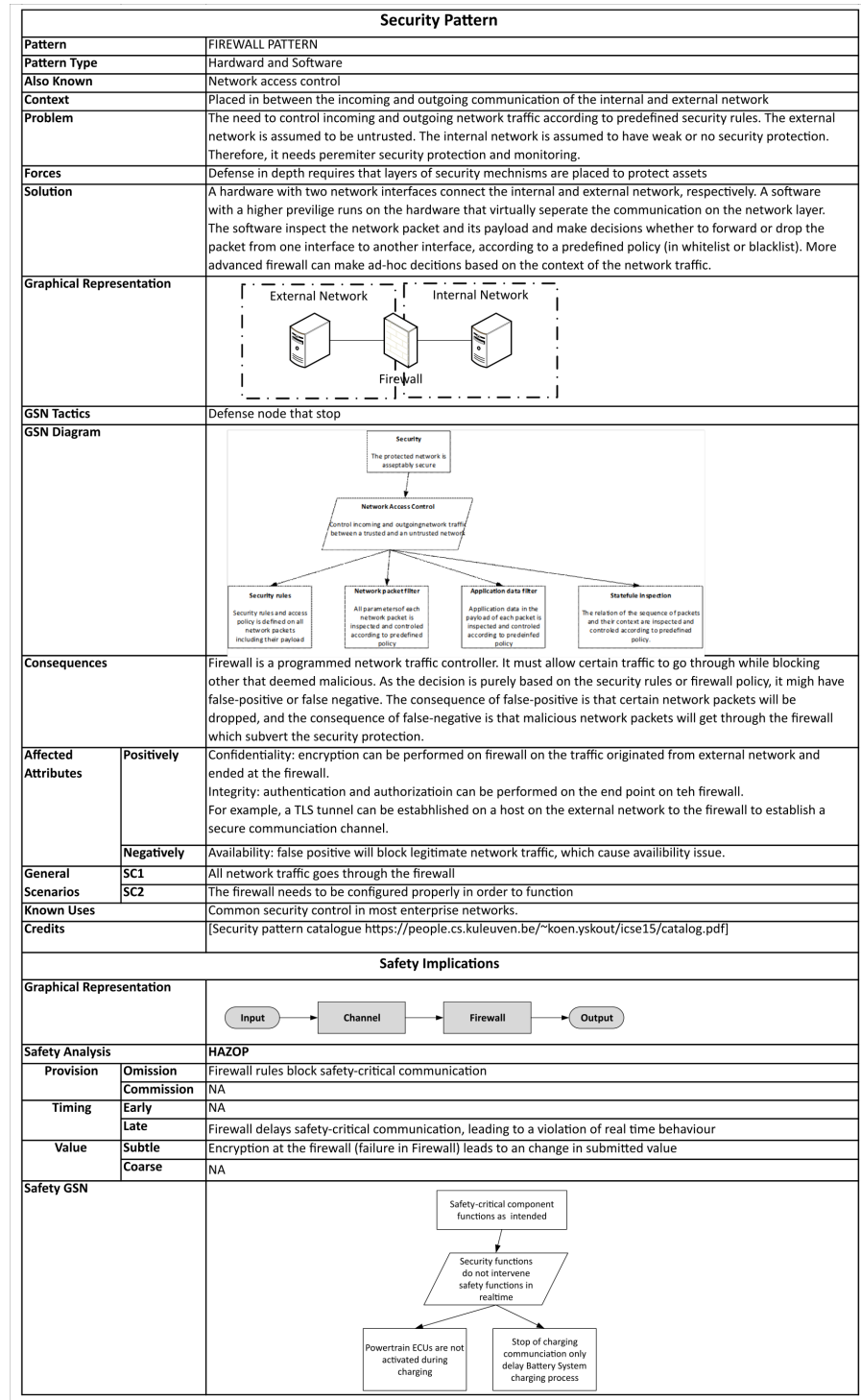

Figure A.11: Security Pattern Example 